\title{
Uncovering Large Groups of Active Malicious Accounts in Online Social Networks
}

\author{
Qiang Cao Xiaowei Yang \\ Duke University \\ \{qiangcao, xwy\}@cs.duke.edu
}

\author{
Jieqi Yu Christopher Palow \\ Facebook Inc. \\ \{jieqi, cpalow\}@fb.com
}

\begin{abstract}
The success of online social networks has attracted a constant interest in attacking and exploiting them. Attackers usually control malicious accounts, including both fake and compromised real user accounts, to launch attack campaigns such as social spam, malware distribution, and online rating distortion.

To defend against these attacks, we design and implement a malicious account detection system called SynchroTrap. We observe that malicious accounts usually perform loosely synchronized actions in a variety of social network context. Our system clusters user accounts according to the similarity of their actions and uncovers large groups of malicious accounts that act similarly at around the same time for a sustained period of time. We implement SynchroTrap as an incremental processing system on Hadoop and Giraph so that it can process the massive user activity data in a large online social network efficiently. We have deployed our system in five applications at Facebook and Instagram. SynchroTrap was able to unveil more than two million malicious accounts and 1156 large attack campaigns within one month.
\end{abstract}

\section{Categories and Subject Descriptors}

C.2.0 [Computer-Communication Networks]: General-Security and protection; K.6.5 [Management of Computing and Information Systems]: Security and protection

\section{General Terms}

Security, Design

\section{Keywords}

Malicious account detection; scalable clustering system; online social networks

\section{INTRODUCTION}

Online social networks (OSNs) such as Facebook, Google+, Twitter, or Instagram are popular targets for cyber attacks. By creating fake accounts $[19,44]$ or compromising existing user accounts $[10$,

Permission to make digital or hard copies of part or all of this work for personal or classroom use is granted without fee provided that copies are not made or distributed for profit or commercial advantage, and that copies bear this notice and the full citation on the first page. Copyrights for third-party components of this work must be honored. For all other uses, contact the owner/author(s). Copyright is held by the author/owner(s).

CCS'14, November 3-7, 2014, Scottsdale, Arizona, USA.

ACM 978-1-4503-2957-6/14/11.

http://dx.doi.org/10.1145/2660267.2660269.
25], attackers can use online social networks to propagate spam messages, spread malware, launch social engineering attacks, or manipulate online voting results.

Much of the previous work in defending against these attacks [1$3,35]$ aims to directly identify the fake or compromised accounts an attacker controls. There exist two broad approaches. One approach is to use an account's social network connectivity $[19,41,45,46]$ to infer whether it is fake or not. This approach can help uncover fake accounts that have few connections to the real social network, but cannot reliably identify compromised real user accounts or well-maintained fake accounts that have acquired many social connections [44]. Another approach, widely adopted in practice $[20,35,47]$, is to build machine learning classifiers to infer malicious (fake or compromised) accounts. This approach can effectively classify those malicious accounts with a set of known malicious features, but may miss many malicious accounts with unknown features.

Motivated by the above challenges, Wang et al. [42] and Beutel et al. [16] have explored a new approach to uncover malicious accounts. They analyzed the aggregate behavioral patterns of social network accounts to distinguish malicious accounts from legitimate ones. In particular, Wang et al. analyzed how the http requests from fake accounts differ from those from real user accounts and used this feature to identify fake accounts. Beutel et al. showed that malicious accounts tend to post fake likes to fraudulent Facebook pages at roughly the same time, and designed CopyCatch to detect such synchronized posts.

This work advances the state of the art of using aggregate behavioral patterns to uncover malicious accounts. Motivated by CopyCatch, we show that malicious accounts tend to act together in a variety of social network context. In addition to posting fake likes, they may log on, install social network applications, upload spam photos, and so on in a loosely synchronized manner $(\$ 2)$.

We then present SynchroTrap, a system that can uncover large groups of malicious accounts that act in loose synchrony. We face a number of unique challenges in designing SynchroTrap (§3) and these challenges set SynchroTrap apart from previous work in this area, i.e., CopyCatch and Clickstream [42]. First, unlike CopyCatch, we aim to detect loosely synchronized behavior for a broad range of social network applications. Therefore, we cannot make the assumption that a user can perform a malicious action only once, i.e., a user can like a specific page only once. This difference in goals has greatly increased the algorithmic complexity of SynchroTrap ( $\$ 4$ and $\S 9$ ).

Second, detecting the actions from malicious accounts is a challenging anomaly detection problem. Malicious actions constitute only a small fraction of the total user actions. For instance, Facebook has more than 600 million daily active users [8] and they per- 
form billions of actions everyday [34]. In contrast, the number of malicious accounts involved in an attack campaign is often on the order of thousands. How can we accurately detect such a weak signal from a large amount of noisy data? Third, we aim to deploy our system on real-world online social networks such as Facebook. Therefore, our detection algorithm must be able to process a few terabytes of data on a daily basis, while many of the off-the-shelf anomaly detection algorithms [26] or previous work, such as Clickstream, do not scale to data of this size.

We have developed several simple but pragmatic techniques to address the above design challenges. First, we model the malicious account detection problem as a clustering problem (§ 3.1). We compare pairwise user actions over a certain time period and group those users who take similar actions at roughly the same time into clusters, and mark a cluster whose size exceeds a tunable threshold as malicious. This is because we observe from a real social network that legitimate social network users take diverse actions over time ( $(2)$. Second, to make the clustering algorithm computationally tractable, we further use an attacker's network resource constraint, e.g., the number of IP addresses under his control, or the attacker's target, e.g., a fraudulent Instagram account, to reduce the pairwise comparison to be per IP address and/or per targeted object, depending on the specific application context. Finally, we partition user action data into small daily or hourly chunks. We design algorithms to aggregate the comparison results between those small chunks to detect malicious actions over a longer period such as a week (§ 4.5). This technique enables us to implement SynchroTrap in an incremental-processing fashion, making it practically deployable at large online social networks.

We have deployed SynchroTrap at Facebook and Instagram for over ten months $(\S 7)$. In a detailed study of one-month data $(\S 8.1)$, we observe that it uncovered more than two million malicious accounts and 1156 malicious campaigns. We have randomly sampled a subset of malicious accounts SynchroTrap caught, and asked security specialists to inspect the accuracy of the results. The manual inspection suggests that our system achieves a precision higher than $99 \%$. During the course of its deployment, SynchroTrap on average catches $\sim 274 \mathrm{~K}$ malicious accounts per week. We have also evaluated the performance of SynchroTrap on a 200-machine cluster at Facebook. The performance results show that our system is able to process Facebook and Instagram's user data. It takes a few hours for SynchroTrap to process the daily data and $\sim 15$ hours to process a weekly aggregation job.

Admittedly, strategic attackers may attempt to spread the actions of malicious accounts to evade SynchroTrap's detection. We analyze SynchroTrap's security guarantee and show that SynchroTrap can effectively limit the rate of malicious actions an attacker performs, even if the attacker controls an unlimited number of malicious accounts $(\$ 6)$. In addition, we provide a set of parameters that operators can tune to achieve a desirable trade-off between false positives and false negatives. With a strict setting, SynchroTrap yields a near-zero false positive rate.

In summary, this work makes the following main contributions: - We observe that malicious accounts tend to act together in a variety of social network context (\$2).

- We have designed, implemented, and deployed SynchroTrap. Our design addresses several practical challenges of using loosely synchronized actions to uncover malicious social network accounts, including how to detect such behavior in a variety of social network applications, and among large and noisy data sets ( $\$ 4)$.

- We present a preliminary analysis of the characteristics of the detected malicious accounts. This analysis may provide insight for other feature-based malicious account detection systems ( $\$ 8)$.

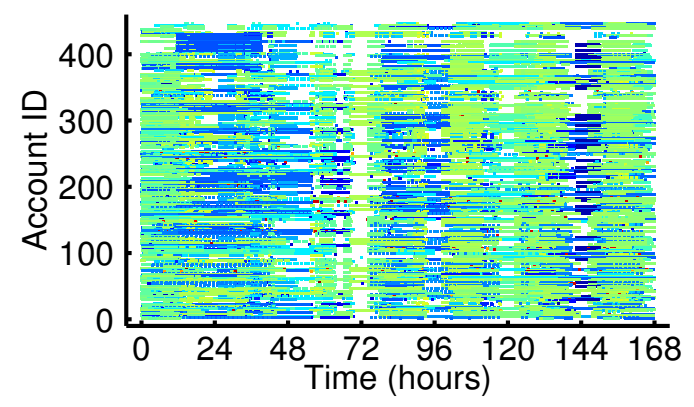

(a) Synchronized attack

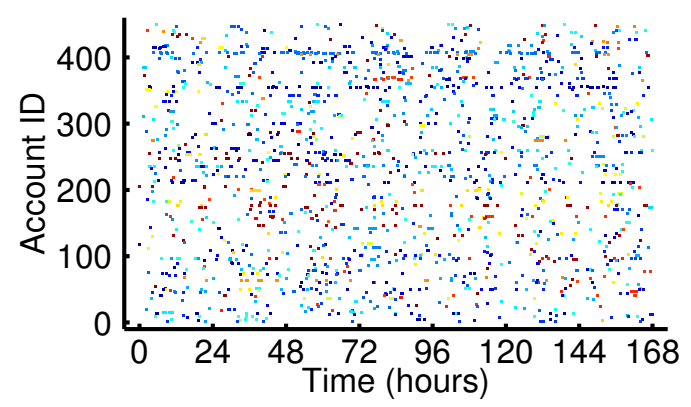

(b) Normal

Figure 1: An example of malicious photo uploads in Facebook. The $x$-axis shows the time when an account uploads a photo, and the $\mathbf{y}$-axis is the account's ID. A dot $(x, y)$ in the figure shows that an account with ID $y$ uploads a photo at time $x$. The color of a dot encodes the IP address of the action. Photo uploads of the same color come from the same IP address.

\section{MOTIVATING EXAMPLES}

In this section, we examine two real-world attack examples that motivate SynchroTrap's design. Beutel et al. [16] observe that malicious accounts post fake likes at around the same time. These two additional examples show that: a) this attack pattern also appears in other social network applications such as Instagram following, and b) malicious accounts not only act together but often from a limited set of IP addresses.

\subsection{Malicious Facebook photo uploads}

Figure 1 compares the photo-uploading activities of malicious users to those of normal users at Facebook. Figure 1(a) plots the photo uploads with timestamps from a group of 450 malicious accounts over a week. Facebook caught those accounts because they promoted diet pills by uploading spam photos. We can see that those accounts use a few IP addresses to upload many spam photos. The horizontal color stripes indicate that they switch among a small set of IP addresses during the one-week period.

Figure 1(b) shows the photo uploads of 450 randomly chosen accounts which have never been flagged as malicious. We refer to those users as normal users. As can be seen, the actions are much more spread out in time and come from a much more diverse set of IP addresses.

\subsection{Inflating followers on Instagram}

Malicious users in Instagram follow target users to inflate the number of their followers. Figure 2 compares user-following activities between 1,000 malicious users and 1,000 normal users. The malicious accounts are sampled from an attack campaign involving $7 \mathrm{~K}$ accounts. 
We can see in Figure 2(a) that those malicious accounts are coordinated to follow a target set of users in batches. The entire group of accounts show a salient on-off action pattern. During the active periods, they follow the same set of users at around the same time. In contrast, normal users exhibit diverse user-following behavior. As shown in Figure 2(b), little perceivable correlation can be found among the user-following sequences of normal users.

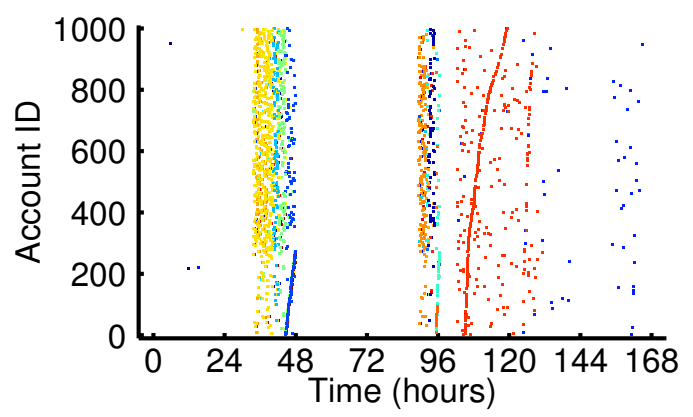

(a) Synchronized attack

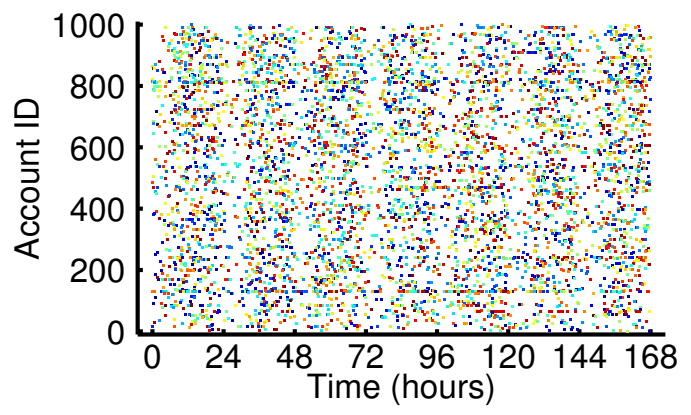

(b) Normal

Figure 2: An example in Instagram user following. The $x$-axis is the timestamp of an account's following action and the $y$ axis is an account's ID. A dot $(x, y)$ shows that an account $y$ follows a targeted account at time $x$. The color of a dot encodes the followed account's ID. Actions of the same color follow the same account.

\subsection{Economic constraints of attackers}

In this subsection, we speculate why various social network attacks tend to happen in loose synchrony. We believe that this is partly due to the economic constraints on the attacker side.

Cost on computing and operating resources. Attackers have limited physical computing resources. Although they can purchase or compromise machines (e.g., botnets), or even rent from cloud computing services, such resources incur financial cost. Furthermore, those computing resources have limited operating time. This is because an infected machine may go offline, recover, or even be quarantined at any time $[32,48]$, and that a machine rental is usually charged based on the consumed computing utility [4]. Another operating cost is the human labor to fabricate fake or compromise real accounts, and to maintain and manage the accounts. Under these operating constraints, an attacker often controls his malicious accounts from a set of machines within a limited time.

Revenue from missions with strict requirements. OSN attackers are often deeply rooted in the underground markets, e.g., BlackHatWorld and Freelancer [33,36,37]. Most of their missions are driven by customer demands with specific requirements. Usually the objective of a campaign is to achieve prevalence in OSNs. There- fore, the mission requirements often include the level of prevalence that a customer pursues and a strict deadline by which the mission must be accomplished. For example, many social-networking tasks in Freelancer solicit X Facebook friends/likes within Y days [33]. Similar tasks target other social network missions, such as followings, posts, reviews, etc. These underground tasks with strict time requirements force attackers to target certain aspects of a victim's service and to act in advance of the mission deadlines.

We call the constraints of limited computing and operating resources as resource constraints, and the constraints of strict requirements on an attacker's missions as mission constraints. Our understanding of these economic constraints and their subsequent manifestation on the activities of controlled accounts helps us directly attack the weak spot of attackers, making it hard for them to evade detection.

\section{SYSTEM OVERVIEW}

\subsection{High-level system architecture}

SynchroTrap is a generic and scalable framework that can effectively throttle large groups of malicious accounts in OSNs. The main idea of SynchroTrap is to use clustering analysis [26] to detect the loosely synchronized actions from malicious accounts at scale. In particular, it measures pairwise user behavior similarity and then uses a hierarchical clustering algorithm [26] to group users with similar behavior over an extended period of time together.

\subsection{Challenges}

We face a number of challenges in making SynchroTrap a practical solution for large-scale OSNs.

Scalability: A main challenge originates from the enormous scale of today's OSNs. First, the large volume of user activity data leads to a low signal-to-noise ratio, making it hard to achieve high detection accuracy. For example, Facebook has more than 600 million daily active users [8], while the number of malicious accounts involved in an attack campaign is often on the order of thousands. As a result, approaches (e.g., clickstream analysis [42]) that use holistic comparison of all user activities may yield low accuracy. In response to this challenge, we partition user actions by OSN applications and detect on a per-application basis $(\S 4.1)$. We further partition user actions by their associated target or source objects, such as IP addresses, followee IDs, and page IDs, to capture the constraints of an attacker ( $(4.2)$.

Second, the sheer volume of activity data prohibits a practical implementation that can cope with generic actions. Large and complex batch computations at Facebook-scale services are prohibitive due to their requirements on hardware capacity (e.g., memory). Such computations make resource sharing difficult and failure recovery costly. To handle massive user activities at Facebook-scale OSNs, we apply divide-and-conquer. We slice the computation of user comparison into smaller jobs along the time dimension and use parallelism to scale $(\S 4.5)$. We then aggregate the results of multiple smaller computations to obtain period-long user similarity.

Accuracy: The diversity of normal user behavior and the stealthiness of malicious activity hinder high accurate detection. Anomaly detection schemes inevitably incur false positives and negatives. As a result, the goal of an automated detection system is often to reduce both the false positive and negative rates. In order to achieve high accuracy, we design SynchroTrap based on our understanding of an attacker's economic constraints. Moreover, as the false positive and false negative rates are usually inversely related, Synchro- 
Trap provides a set of tunable parameters in its design and enables operators to tune these parameters $(\S 4.6)$ for a desired trade-off.

Adaptability to new applications: Attack campaigns can target distinct OSN applications. Because the properties of a user's actions, such as the association between the user and other OSN objects, can vary in different applications, a detection scheme optimized for one application may not be applicable to others. Therefore, it is challenging to develop a generic solution that can adapt to new applications. For example, CopyCatch [16] detects fraudulent page likes (once-only actions), but cannot be used to uncover repeated spam-photo uploads from the same IP addresses. Unlike CopyCatch, in our design we decouple the similarity metrics (\$ 4.3) from the clustering algorithm ( $\$ 4.4)$, which enables us to handle both once-only and other generic actions. Furthermore, we represent an action with a tuple abstraction (\$ 4.2), including a timestamp dimension and an attacker constraint dimension. This abstraction makes the system design independent of the OSN applications that SynchroTrap protects.

\section{SYSTEM DESIGN}

In this section, we describe the design of our system in detail. We categorize user actions according to OSN applications ( $§ 4.1)$ and perform detection on a per-application basis. We define a generic matching metric for time-stamped user actions ( $\$ 4.2)$ and quantify the similarity of a user pair using the fraction of their matched actions ( $\$ 4.3)$. We use a single-linkage hierarchical clustering algorithm to group users based on the pairwise user similarity $(\S 4.4)$. In $\S 4.5$, we parallelize the computation of user-pair comparison to address the large-data challenge.

\subsection{Partitioning activity data by applications}

OSNs usually provide many features and functions in the form of OSN applications, such as photo uploading, page like, messaging, etc. Malicious accounts are not necessarily coordinated across all types of actions allowed by the platforms. To reduce operational cost, an attacker can focus his missions and target only partial dimensions of the user action space, e.g., uploading spam photos, promoting rogue apps, etc. As a result, a scheme using holistic comparison of user activities may miss malicious users that target only particular OSN functions. This problem is reminiscent of the "curse of dimensionality" in clustering high-dimensional data [29].

To mitigate the impact of irrelevant actions, we categorize a user's actions into subsets according to the applications they belong to, which we call application contexts. We then detect malicious accounts within each application context. For example, we separate the photo upload and page like applications to suppress spam photos and fraudulent page likes, respectively. Next, we describe how we cluster user actions for an OSN application.

\subsection{Comparing user actions}

In SynchroTrap, we abstract time-stamped user actions as tuples, each of which has an explicit constraint field that can express both resource and mission constraints. We require exact match on the constraint field to capture an attacker's constraints. From the point of view of an OSN provider, each user action has a number of attributes. Table 1 summarizes the main attributes used in this paper and their definitions. An AppID can include an identifier of an application-layer network protocol (e.g., HTTP) to indicate a fine-grained application category. An AssocID can be the identifier of an associated OSN object (e.g., photos, pages, users, etc). We denote our tuple abstraction of a time-stamped user action as $\langle U, T, C\rangle$, where $U, T$, and $C$ represent user ID, action timestamp, and a constraint object, respectively. A constraint object can be a combination of certain action attributes, such as a concatenation of AssocID, source IP address, etc.

\begin{tabular}{l|l} 
Attribute & Meaning \\
\hline UID & User ID \\
\hline Timestamp & Timestamp of the user action \\
\hline AppID & Application identifier, e.g., posting and messaging \\
\hline AssocID & Object ID with which a user action is associated \\
\hline IP address & IP address of the user client
\end{tabular}

Table 1: Attributes of a user action and their meanings.

Our tuple abstraction of user actions is expressive. It enables SynchroTrap to quickly adapt to a specific attack in an application, provided that the constraint field is properly designated. For example, one can choose the followee identifier (a type of AssocID) as the constraint field to defeat abusive user following on Instagram.

Based on the tuple abstraction, we define action match, denoted by " $\approx$ ". Two actions of different users match if they pertain to the same constraint object and their timestamps fall into the same time window of a pre-defined length $T_{\text {sim }}$ (e.g., 1 hour). That is, a match of two user actions is possible only if they occur within a matching window of $T_{\text {sim }}$.

$\left\langle U_{i}, T_{i}, C_{i}\right\rangle \approx\left\langle U_{j}, T_{j}, C_{j}\right\rangle$ if $C_{i}=C_{j}$ and $\left|T_{i}-T_{j}\right| \leq T_{s i m}$

\subsection{Pairwise user similarity metrics}

We quantify the similarity of two users by computing the fraction of their matched actions during a time period $T_{p}$ (e.g., a week). We use the Jaccard similarity, a widely-used metric that measures the similarity of two sets [24], as the similarity metric. The Jaccard similarity metric ranges from 0 to 1 . A value close to 1 indicates high similarity.

Per-constraint similarity. We introduce the per-constraint similarity to measure the fraction of matched actions on a single constraint object (e.g., a single source IP address). Let $A_{i}$ be the set of actions performed by user $U_{i}$, i.e. $A_{i}=\left\{\langle U, T, C\rangle \mid U=U_{i}\right\}$. As we require exact match on the constraint field of user actions, we further break down $A_{i}$ into disjoint subsets according to the value of the constraint field, i.e., where $A_{i}^{k}=\left\{\langle U, T, C\rangle \mid U=U_{i}, C=C_{k}\right\}$. We derive user similarity on a single constraint object using Jaccard similarity, as shown below. When we compute the Jaccard similarity, we apply the action matching operator " $\approx "(\S 4.2)$ to obtain the set intersection and the set union.

$$
\operatorname{Sim}\left(U_{i}, U_{j}, C_{k}\right)=\frac{\left|A_{i}^{k} \cap A_{j}^{k}\right|}{\left|A_{i}^{k} \cup A_{j}^{k}\right|}
$$

Overall similarity. In certain OSN applications, the association of a user to a constraint object does not appear more than once. For example, in Facebook app installation, a user can install an app only once. In such cases, the Jaccard similarity of a user pair on a single constraint object (i.e., an app ID) can only be either 0 or 1. To better characterize the similarity among users, we use the overall Jaccard similarity, which accounts for user actions across all constraint objects.

$$
\operatorname{Sim}\left(U_{i}, U_{j}\right)=\frac{\left|A_{i} \cap A_{j}\right|}{\left|A_{i} \cup A_{j}\right|}=\frac{\sum_{k}\left|A_{i}^{k} \cap A_{j}^{k}\right|}{\sum_{k}\left|A_{i}^{k} \cup A_{j}^{k}\right|}
$$

\subsection{Scalable user clustering}

We choose the single-linkage hierarchical clustering algorithm [26] to cluster users due to its effectiveness and potential scalability. We 
Edge similarity threshold

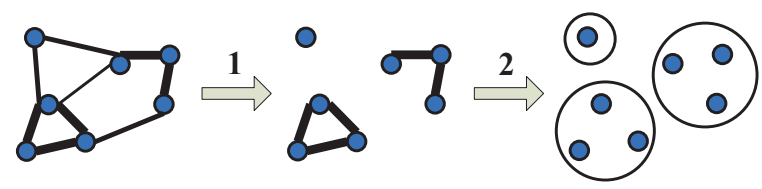

Figure 3: Transforming the single-linkage hierarchical clustering algorithm to the algorithm of connected components in two steps. Edges represent similarity between users. A user pair connected by a thicker edge has a higher similarity.

do not use other off-the-shelf clustering schemes because they either rely on a special distance metric (e.g., Euclidean distance in k-means), or are not scalable. We refer readers to [26] for a complete review of the clustering techniques. In addition, we do not seek to use graph partitioning algorithms for clustering users, because even widely-used graph partitioning tools like METIS [28] take many hours to process a graph with only multiple millions of nodes [40]. Instead, our objective is to transform our detection scheme to a clustering algorithm that can scale up to large OSNs.

Single-linkage hierarchical clustering. The single-linkage hierarchical clustering algorithm uses an agglomerative approach that begins with each user as a different cluster, and iteratively merges clusters with high similarity and produces larger clusters. This algorithm generates a cluster-merging dendrogram that shows the merging hierarchy of clusters and the degree of similarity on each level. By breaking the dendrogram at a desired level, one obtains a set of clusters in which intra-cluster user similarity exceeds a certain threshold. A detailed description of the algorithm is documented in [26]. Because this algorithm relies on a sequential process to construct the entire dendrogram in a bottom-up fashion, a straightforward implementation is difficult to scale.

Making the algorithm suitable for parallel implementation. The key property of single-linkage hierarchical clustering is that the similarity of two clusters is determined by the maximum similarity among all pairs of users drawn from each different cluster. The cluster-similarity metric merges a group of close clusters in each iteration into a larger connected component in a user similarity graph, where nodes are users and an undirected edge exists between a pair of users if their similarity is above a certain threshold.

Using this property we adapt the single-linkage hierarchical clustering algorithm to a parallel version. Our idea is that if we set the similarity threshold first and filter out user pairs below that, the desired user clusters are exactly the connected components in the pruned user similarity graph. Therefore, we can employ an efficient graph algorithm [27] to search for connected components. Figure 3 illustrates our two-step adaptation of the single-linkage clustering algorithm. We choose to adapt to the connected components algorithm because it is highly scalable on massive graphs due to its inherent parallelism [27].

User-pair filtering function. We use a filtering function to select user pairs with action similarity above a certain degree. We introduce two criteria to choose a user pair according to their similarity at different granularities ( $(4.3)$.

- F1: There exists at least one constraint object, for which users have a per-constraint similarity above a certain threshold.

- F2: Their overall similarity is above a certain threshold.

The first filtering criterion uncovers malicious user pairs that manifest loosely synchronized behavior on a set of single constraint

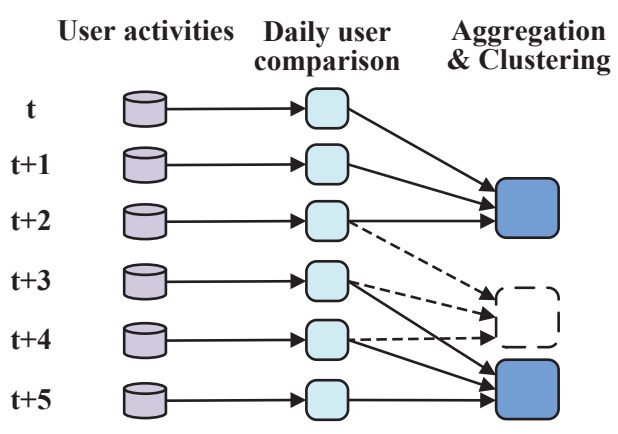

Figure 4: SynchroTrap's processing pipeline at Facebook. A new aggregation job (dashed) does not incur re-execution of daily jobs. Arrows indicate the data flow.

objects (e.g., IP addresses). In some cases, malicious accounts may even spread their actions over a number of constraint objects. We use criterion $F 2$ to compare user similarity for applications where a user can carry out a certain action only once per constraint object.

\subsection{Parallelizing user-pair comparison}

To process continuous user-activity data stream at scale, we use incremental processing. In particular, we divide the large computation of user-pair comparison on a bulk data set into a series of smaller ones in the time dimension. We store the intermediate results and aggregate them over a certain time period. This processing pipeline greatly reduces the size of a single job and thus its hardware consumption, making SynchroTrap a more scalable and manageable solution in practice.

\subsubsection{Daily comparison}

Figure 4 shows the data flow of SynchroTrap's processing pipeline at Facebook. We slice the computation of user comparison and designate daily jobs to generate similar user pairs based on the useractivity log. Because SynchroTrap detects consistently loosely synchronized activities over a sustained period of time, we aggregate daily similarity metrics and perform user clustering periodically (e.g., weekly). As shown in Figure 4, because aggregation jobs can reuse the results of daily jobs, a new aggregation job does not incur re-execution of daily jobs.

We design an aggregatable data interface between daily jobs and aggregation jobs by decomposing the period-long user similarity $(\S 4.3)$ over days, as shown below. Let $A_{i, t}^{k}$ denote the set of actions on constraint object $C_{k}$ that user $U_{i}$ performs on day $t$, i.e. $A_{i, t}^{k}=\left\{\langle U, T, C\rangle \mid U=U_{i}, C=C_{k}, T\right.$ is within day $\left.t\right\}$. For a user pair $\left(U_{i}, U_{j}\right)$ and a constraint object $C_{k}$, we generate and store the number of their daily action matches, $\left|A_{i, t}^{k} \cap A_{j, t}^{k}\right|$, and the number of daily total actions that each of them has carried out, i.e., $\left|A_{i, t}^{k}\right|$ and $\left|A_{j, t}^{k}\right|$.

$$
\begin{aligned}
\operatorname{Sim}\left(U_{i}, U_{j}, C_{k}\right) & =\frac{\left|A_{i}^{k} \cap A_{j}^{k}\right|}{\left|A_{i}^{k} \cup A_{j}^{k}\right|}=\frac{\left|A_{i}^{k} \cap A_{j}^{k}\right|}{\left|A_{i}^{k}\right|+\left|A_{j}^{k}\right|-\left|A_{i}^{k} \cap A_{j}^{k}\right|} \\
& =\frac{\sum_{t}\left|A_{i, t}^{k} \cap A_{j, t}^{k}\right|}{\sum_{t}\left|A_{i, t}^{k}\right|+\sum_{t}\left|A_{j, t}^{k}\right|-\sum_{t}\left|A_{i, t}^{k} \cap A_{j, t}^{k}\right|}
\end{aligned}
$$

By aggregating the daily results, we derive user similarity over a course of time. The last equality holds because user-action matches across days are rare, as the size of a matching window we choose is on the order of minutes or a few hours. 


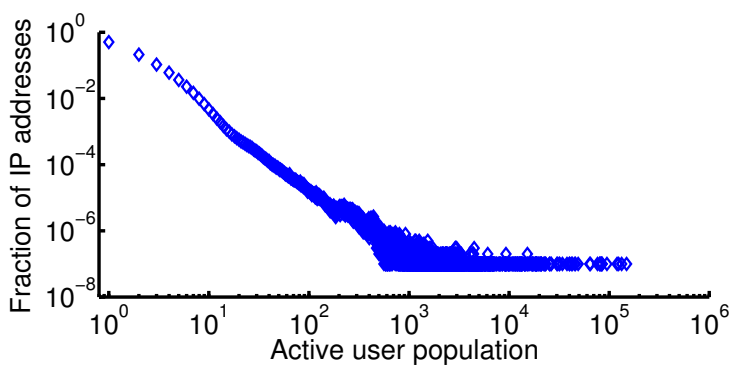

Figure 5: Distribution of user population over IP addresses at Facebook login. We depict the fraction of IP addresses with respect to the number of active users per IP address.

\subsubsection{Hourly comparison with sliding windows}

Stragglers in daily jobs. A straightforward MapReduce implementation of the daily user comparison yields jobs whose completion time could be prolonged by straggler reducers. This straggler issue is caused by the highly skewed distribution of user actions over constraint objects. Even on a daily basis, there exist hot objects that are associated with an extremely large number of actions/users. Figure 5 shows the distribution of the number of login users over IP addresses on one day at Facebook. As we can see, while most of IP addresses are not used by many users (less than 100), the user population distribution per IP is heavy-tailed. These few popular IP addresses used by more than $100 K$ daily active users can lead to straggler reducers that might run for days.

Mitigation with overlapping sliding windows. We mitigate this issue by further partitioning users with their actions on large constraint objects. If the number of users in a partition is reduced by a factor of $\frac{1}{f}(f>1)$, the execution time can be reduced by $\frac{1}{f^{2}}$, as we perform a quadratic number of user-user similarity computations. The challenge is to effectively partition the user activity data, while retaining the capability of accurately capturing action matches. Our insight is to divide the user actions into overlapping sliding windows in the time dimension and to process different sliding windows in parallel. This method not only mitigates the straggler issue by feeding each individual worker smaller chunks of data, but also effectively filters out unmatched actions that reside in different sliding windows before the pairwise comparison.

Figure 6 illustrates how we partition by overlapping sliding windows to precisely capture all possible user-action matches with a matching window set to $T_{\text {sim }}$. In principle, a sliding window size $>T_{\text {sim }}$ and an overlapping period $\geq T_{\text {sim }}$ can guarantee the full coverage of user-action matches. This is because a sliding window size $>T_{\text {sim }}$ ensures that any user-action match with a maximum spanning period $T_{\text {sim }}$ can be covered by a sliding window; an overlapping period $\geq T_{\text {sim }}$ ensures that the sliding windows are dense enough to cover the all user-action matches across windows.

Sliding window setup. Counting in each overlapping sliding window entails duplicates of user-action matches that appear in the overlapping area. The de-duplication of action matches could be complicated if the sliding window size and the overlapping period are not properly set. To strike an appropriate balance between the effectiveness of cutting the data volume within each sliding window and the complexity of de-duplicating action matches, we choose to use a sliding window size of $2 T_{\text {sim }}$ and an overlapping period of length $T_{\text {sim }}$ (Figure 6). With such a setting, we achieve single counting by simply discarding the user action matches within the second (or the first) half of each sliding window.

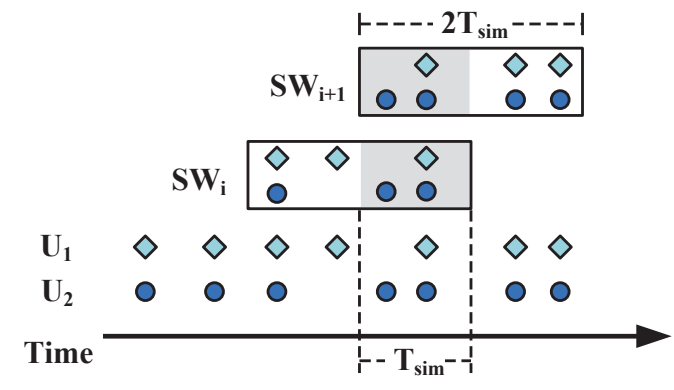

Figure 6: Settings of the overlapping sliding windows in SynchroTrap. Action matches within the overlapping area (shaded) are exactly double counted. We depict actions from different users using different markers.

We now discuss our parallel counting scheme's guarantee that one can exactly single count all user-action matches by independently counting in each sliding window only. Suppose we have a sequence of sliding windows $S W_{i}=\left[i T_{\text {sim }},(i+2) T_{\text {sim }}\right)(i \geq 0)$. Without loss of generality, let $t_{1}$ and $t_{2}$ be the timestamps of two matched user actions, where $t_{2} \in\left[t_{1}, t_{1}+T_{\text {sim }}\right]$. Suppose $t_{1} \in$ $\left[j T_{\text {sim }},(j+1) T_{\text {sim }}\right)(j \geq 0)$. We have $t_{2}<(j+2) T_{\text {sim }}$. Two cases exist regarding the location of the action pair: a) $t_{2}<(j+$ 1) $T_{\text {sim }}$. Both $t_{1}$ and $t_{2}$ belong to the interval $\left[j T_{\text {sim }},(j+1) T_{\text {sim }}\right)$, which is the overlapping area of two consecutive sliding windows $S W_{j-1}$ and $S W_{j}$. Because we discard action matches within each second-half window, the action pair is only single counted in $S W_{j}$; b) $t_{2} \in\left[(j+1) T_{\text {sim }},(j+2) T_{\text {sim }}\right)$. Only $S W_{j}$ covers both $t_{1}$ and $t_{2}$, because $t_{1} \in\left[j T_{\text {sim }},(j+1) T_{\text {sim }}\right)$. Hence the action pair is single counted in $S W_{j}$. We always append an empty half window after the last sliding window in order to cope with the extreme case at the end of the data stream.

\subsection{Improving accuracy}

It is challenging for a detection system, such as SynchroTrap, to achieve desired accuracy for several reasons. First, the volumes and synchronization levels of malicious actions vary in different OSN applications. In extreme cases, attackers may change their strategies over time to evade an existing detection scheme. Second, as a system that influences user experience on an OSN, SynchroTrap must use conservative parameters to minimize the false positive rate, i.e., not flagging any legitimate user as malicious.

SynchroTrap allows OSN operators to tune a set of parameters to achieve the desired trade-off between false positives and false negatives. The main parameters include the action-matching window size $T_{\text {sim }}$ and the filtering thresholds for per-constraint similarity $\left(\operatorname{Sim}_{p c}\right)$ and overall similarity $\left(\operatorname{Sim}_{\text {overall }}\right)$. The settings of these parameters have monotonic effects on false rates: a larger action-matching window enables SynchroTrap to find a larger set of matched actions for two users, and hence increases their similarity on a constraint object; on the other hand, a larger user similarity threshold decreases the number of user pairs considered similar and reduces the likelihood that two users are clustered together. These monotonic effects simplify the process of setting parameters and reduce the need for human intervention. The operators of our system can choose to tune parameter values up or down according to the false positive rate with the current settings. At Facebook we set parameters equal to the values that meet the internal production requirements. We do not reveal the specific parameter settings due to confidentiality agreements. 


\subsection{Computational cost}

In theory, SynchroTrap's computational cost is $O\left(r n^{2}\right)$, where $n$ is the number of daily active users per application and $r$ is the number of daily actions per user. In practice, we can significantly reduce this computational cost because we only need to compare user actions pertaining to the same target object or coming from the same source object. Therefore, in our implementation, the daily computational cost is the $O\left(\mathrm{rm}^{2}\right)$, where $m$ is the number of daily active users per application per target or source object (i.e., per campaign target or per IP address). The cost for weekly aggregation is linear to the number of user pairs generated by daily jobs. The cost for searching connected components in a user similarity graph is $O(n)$. Thus the overall computational cost is $O\left(\mathrm{rm}^{2}+n\right)$.

\section{IMPLEMENTATION}

We built SynchroTrap on top of the Hadoop MapReduce stack [38] at Facebook. We implemented the daily user comparison module and the weekly aggregation module on Hadoop [6], and the clustering module on Giraph [5], a large-graph processing platform based on the Bulk Synchronous Parallel (BSP) model [30]. Giraph provides a parallel implementation of the connected components algorithm. Apart from the basic functions supported by Facebook's infrastructure, our implementation of SynchroTrap consists of 2,500 lines of Java code and 1,500 lines of Python code.

\section{SECURITY ANALYSIS}

In this section we provide a security analysis of our approach under various adversarial strategies.

Spread-spectrum attacks. Attackers could attempt to hide the synchronization signal that SynchroTrap detects, which we call the spread-spectrum attacks. Given a target amount of abusive actions, attackers can statistically spread actions over either a longer time period or more constraint objects (e.g., IP addresses and campaign targets). Due to the increased resource cost and the reduced campaign revenue, such attacks are less profitable. We now show that SynchroTrap limits the damage of attack campaigns, even if attackers control an unlimited number of accounts. We provide an upper-bound analysis on the total number of actions that attackers can perform on a constraint object during a certain period of time.

Suppose our detection window $T_{p}$ (e.g., one week) contains $w$ action-matching windows of length $T_{m}$ (e.g., 1 hour). Because per-account rate-limiting is widely used in OSNs such as Facebook $[13,14]$, we assume that an account can perform at most $L$ actions within each action-matching window. Although the number of each account's actions is bounded by $w L$, without SynchroTrap the total malicious actions remain unlimited if attackers can control an extremely large number of malicious accounts.

In contrast, SynchroTrap limits the total number of abusive actions on a constraint object (e.g., an IP address), irrespective of the number of malicious accounts an attacker controls. The intuition is that under SynchroTrap an attacker has to spread out the actions of his accounts over matching windows so that a pair of accounts do not have many matched actions. Therefore, given $w$ matching windows, the number of malicious accounts that can simultaneously act on a constraint object is bounded.

Specifically, SynchroTrap uses the Jaccard similarity to evaluate the action sets of two users. In order to evade the detection, the fraction of matched actions of malicious accounts $U_{i}$ and $U_{j}$ must be below a certain threshold $p(0<p<1)$ : $\left|A_{i} \cap A_{j}\right| \leq p \times\left|A_{i}\right|$ and $\left|A_{i} \cap A_{j}\right| \leq p \times\left|A_{j}\right|$. An optimal attack strategy is to schedule a group of accounts according to the set of such action sets $\left\{A_{i}\right\}$ that has the maximum cardinality so as to minimize the chances two malicious accounts are caught in the same cluster. Finding $\left\{A_{i}\right\}$ with the maximum cardinality is still an open problem in intersection set theory [18], which poses a challenge to attackers.

We give an upper bound on the cardinality of such a set $\left\{A_{i}\right\}$ by computing the maximum size of its superset. We find such a superset $\left\{B_{i}\right\}$ in which $B_{i} \subseteq B_{j}$ only if $B_{i}=B_{j}$. That is, in $\left\{B_{i}\right\}$ none of the sets is contained in another. Because set $\left\{B_{i}\right\}$ does not require a threshold on $\left|B_{i} \cap B_{j}\right|$, it relaxes the conditions of set $\left\{A_{i}\right\}$ and hence $\left\{A_{i}\right\} \subset\left\{B_{i}\right\}$. Set $\left\{B_{i}\right\}$ approximates set $\left\{A_{i}\right\}$ if the matched fraction threshold $p$ is set close to 1 . In set theory, $\left\{B_{i}\right\}$ is called an antichain of sets in which none of the sets is a subset of another. According to the Sperner's theorem [15], given that the detection window contains $w$ matching windows, the size of the maximum antichain satisfies $\left|\left\{B_{i}\right\}\right| \leq\left(\begin{array}{c}w \\ \lfloor/ 2\rfloor\end{array}\right)$. Therefore, we have $\left|\left\{A_{i}\right\}\right|<\left(\begin{array}{c}w \\ \lfloor/ 2\rfloor\end{array}\right)$, which specifies the upper bound of the number of active malicious accounts per constraint object. Thus, the total number of actions from this malicious account group is further bounded by $\left(\begin{array}{c}w \\ \lfloor w / 2\rfloor\end{array}\right) w L$, assuming all of the accounts are kept active during the detection window $T_{p}$.

Aggressive attacks. Aggressive attacks could be launched by controlling accounts to perform bulk actions within a short time period. SynchroTrap may miss such attacks if the user action-set size or the user-pair similarity does not meet the criteria of SynchroTrap's user-pair filtering function. However, such attacks have been the focus of existing countermeasures [35], which look for the abrupt changes in user activity. Our system works together with existing anomaly detection schemes and complements them by targeting the stealthier attacks.

\section{DEPLOYMENT}

We deployed SynchroTrap at Facebook and Instagram to uncover malicious accounts and integrated it into the site-protecting stack at Facebook. In this section, we present five use cases (\$ 7.1) and describe how the findings of SynchroTrap can be used to better monitor and protect OSN services (§ 7.2).

\subsection{Use cases at Facebook and Instagram}

We present SynchroTrap's use cases according to the constraint by which an attack campaign is bound. For each type of attackerside constraint, we present a couple of use cases at Facebook and Instagram.

Resource-constrained synchronization. The resource constraint we use is the source IP addresses from which the attacks originate. We deployed SynchroTrap with this configuration at Facebook user login and photo upload. An OSN provider could also include certain security cookies $[7,12]$ into SynchroTrap's constraint field, which enables the detection of resource-constrained attacks at a finer granularity.

Mission-constrained synchronization. The mission constraints we use are target object IDs, which include Facebook app ID, Facebook page ID, and Instagram followee ID as the constraint field, respectively. We deployed SynchroTrap at Facebook app installation and page like, and at Instagram user following context. We used the overall similarity in these cases.

\subsection{Signatures and response}

As an unsupervised detection scheme, SynchroTrap automatically discovers large groups of malicious accounts after its deployment. This malicious account corpus can be used as high-quality training data to build accurate classifiers. We now describe how 
we fingerprint attacks and take actions on detected accounts and user-created content.

Attack signatures. SynchroTrap extracts the common constraint objects on which groups of suspicious accounts act together. The OSN entities pointed by those constraint objects can be abusive, and thus can be used as attack signatures. They include rogue Facebook apps, Facebook pages with inappropriate content, abusive Instagram accounts soliciting excessive followers, etc. By tracking back to the complete user action log, SynchroTrap can even provide the fingerprints of an attacker's machines, including IP addresses, user agents, browser cookies, etc. All of the above signatures can be used to build fast classifiers to suppress future attacks in nearly real time [35], and to decide on proper responses.

Response. The response to attacks in SynchroTrap is multifold: large groups of detected accounts are challenged with CAPTCHAs; actions performed in attack campaigns are invalidated in retrospect; and user-created content, such as photos, is sent for automated sanity check (e.g., photoDNA [9]) or manual inspection.

\section{EVALUATION}

We evaluate SynchroTrap using a one-month execution log at Facebook in August 2013. We answer the following questions to show that SynchroTrap provides a practical solution for large online social networks:

- Can SynchroTrap accurately detect malicious accounts while yielding low false positives?

- How effective is SynchroTrap in uncovering new attacks?

- Can SynchroTrap scale up to Facebook-size OSNs?

We obtain SynchroTrap's detection accuracy by manually inspecting sampled accounts and activities it uncovered. We then study the new findings through cross-validation against existing approaches that run at Facebook. We examine the social connectivity of the identified accounts by using SybilRank [19], a scalable social-graph-based fake account detection system. We also share the operation experience to shed light on how SynchroTrap works in practice over time. Lastly, we demonstrate the scalability of SynchroTrap using performance measurements obtained from a 200-machine cluster.

\subsection{Validation of identified accounts}

We first validate the malicious accounts with support from the Facebook security team. We proceed with investigation of the confirmed accounts to understand how adversaries managed to take control of them. Furthermore, we study the network-level characteristics of the detected attacks, including the email domains and IP addresses used by malicious accounts.

\begin{tabular}{l|l|l|l|l|l} 
Application & $\begin{array}{l}\text { Page } \\
\text { like }\end{array}$ & $\begin{array}{l}\text { Instagram } \\
\text { follow }\end{array}$ & $\begin{array}{l}\text { App } \\
\text { install }\end{array}$ & $\begin{array}{l}\text { Photo } \\
\text { upload }\end{array}$ & Login \\
\hline Campaigns & 201 & 531 & 74 & 29 & 321 \\
\hline Accounts & $730 \mathrm{~K}$ & $589 \mathrm{~K}$ & $164 \mathrm{~K}$ & $120 \mathrm{~K}$ & $564 \mathrm{~K}$ \\
\hline Actions & $357 \mathrm{M}$ & $65 \mathrm{M}$ & $4 \mathrm{M}$ & $48 \mathrm{M}$ & $29 \mathrm{M}$ \\
\hline Precision & $99.0 \%$ & $99.7 \%$ & $100 \%$ & $100 \%$ & $100 \%$
\end{tabular}

Table 2: Identified accounts and precision. Precision is the portion of identified accounts that are confirmed malicious. We derived precision from manual inspection of randomly sampled accounts by the Facebook security team.

Methodology. A main challenge to validate the detected accounts and their actions is their large number. During the month of our

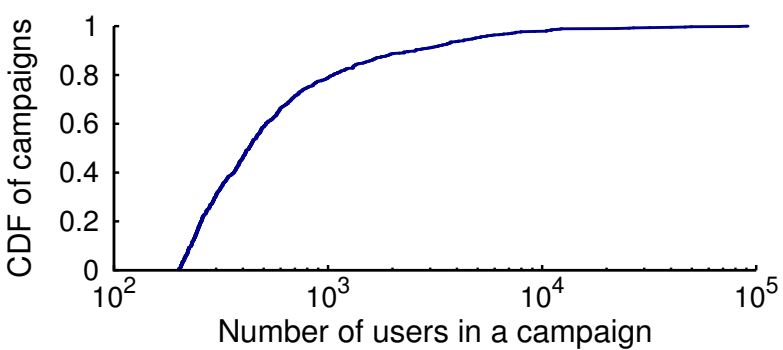

Figure 7: CDF of campaigns with respect to the number of involved users. In a large campaign, an attacker manipulates multiple thousands of malicious accounts.

study, SynchroTrap uncovers millions of accounts. Manually reviewing all those accounts imposes prohibitive human workload. Furthermore, cross validating the detected accounts with other existing Facebook countermeasures is not possible because a large fraction of detected accounts are not caught by other methods ( $\$ 8.2$ ). Therefore, our approach is to inspect representative samples of the detected accounts with manual assistance from the security specialists. We randomly sample subsets of the detected accounts for inspection and obtain the false rates.

Precision. Table 2 shows the numbers of suspicious accounts SynchroTrap caught and attack campaigns uncovered by SynchroTrap, and the precision in each application. In total, SynchroTrap detected 1156 large campaigns that involve more than 2 million malicious accounts, with a precision higher than $99 \%$. Table 2 also shows that the large attack campaigns are comprised of millions of user actions. Among the five deployed applications, attackers were more active in page like and user following, presumably because campaigns in these applications are more lucrative. By uncovering large campaigns, SynchroTrap allows Facebook and Instagram to identify and properly invalidate millions of malicious user actions in each application.

Post-processing to deal with false positives. False positives are detrimental to OSN user experience. Besides adding human efforts into the process of setting parameters $(\$ 4.6)$, we further reduce false positives through post-processing. First, we discard small user clusters and screen out only large clusters, which are more likely to result from large attacks. Based on the experience with the system, the Facebook security team sets a threshold of 200, above which almost all users in each cluster are found malicious. Second, we do not invalidate all actions that a malicious account has performed during a detection window $T_{p}$, but conservatively focus on those that match at least one action of each of the other accounts in the same cluster. This post processing step helps rule out valid actions that a user account may have delivered while being compromised.

Scale of campaigns. Figure 7 shows the CDF of the scale of the attack campaigns after post-processing, in terms of the number of involved malicious accounts. While $80 \%$ of the campaigns involve fewer than 1,000 accounts, we also find a few very large campaigns, in which attackers manipulate a few thousands of accounts.

How are the malicious accounts taken under control? Because attackers have to use accounts to perform malicious activities in OSNs, it is critical for them to own or hijack a large number of accounts before launching their campaigns. To understand how adversaries take control of accounts, the Facebook security team classifies the reviewed accounts into categories based on how they were involved in campaigns. The means by which attackers harness 


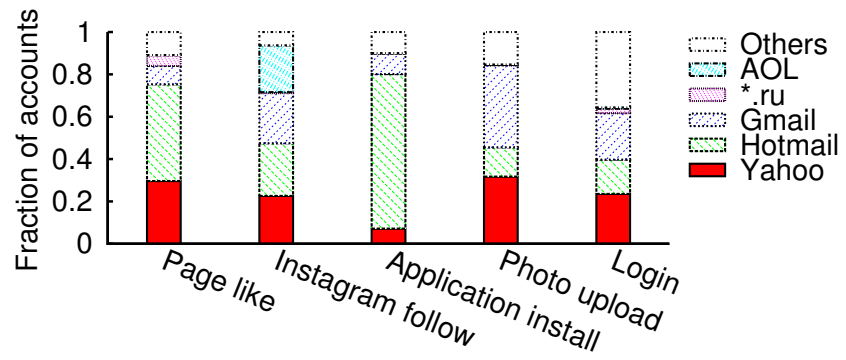

Figure 8: Breakdown of top email domains associated to the malicious accounts in each application.

accounts include creating fake accounts with fraudulent user information [19,44], compromising user accounts via malware [10], stealing user credentials by social engineering [17, 25], etc. A breakdown of the malicious accounts in app installation is shown in Table 3. In this application, attackers manipulate malicious accounts to promote rogue Facebook apps that can later be used to send out spam, to steal user personal information, etc. Clearly, fake accounts, social engineering, and malware are the dominant malicious account sources, accounting for more than $90 \%$ of the detected accounts.

\begin{tabular}{c|c|c|c} 
Fake accounts & Social Engineering & Malware & Others \\
\hline $28.6 \%$ & $21.4 \%$ & $42.9 \%$ & $7.1 \%$
\end{tabular}

Table 3: Classification of the malicious accounts detected at Facebook app install.

Network-level characteristics. We study the email domains and IP addresses used by malicious users to shed light on the networklevel characteristics of attacks.

An OSN account usually associates to a contact email address. Figure 8 shows the distribution of the email domains of the identified accounts in each application. As we can see, the email credentials used by the controlled accounts are mainly from five domains, including those major email domains Yahoo, Hotmail, and Gmail. Email domains with accounts that can be obtained from underground markets (e.g., Yahoo, Hotmail, and AOL) are likely to be used to provide fraudulent contact email addresses for controlled accounts. Whereas Gmail accounts incur higher cost to attackers according to an underground market survey [37], a fraction of the identified accounts are found to use Gmail addresses. In addition, a non-negligible fraction of the contact email addresses are from the domain *.ru, which is dominated by mail.ru and yandex.ru. Because the identified accounts used a diverse set of email addresses, this result suggests that the email domain alone is not a reliable criterion to detect malicious accounts.

We further study the source IP addresses of the detected malicious activities. We found that the two million detected accounts have used $\sim 1.2$ million IP addresses in total. Figure 9 shows the distribution of the IPv4 addresses used by attackers in each application. As can be seen, the threats are initiated from three major regions of the IPv4 address space: 36.67.* - 44.99.*, 77.246.*_ 125.226.*, and 170.226.* - 207.78.*. The distributions of IP addresses in different applications are close to each other, except that attackers in app install use more intensively the IP addresses from the region $77.246 . *-125.226{ }^{*}$. We investigate a random sample set of those IP addresses via queries to WHOIS servers, which provide the registration information of the domain names. Many IP ad-

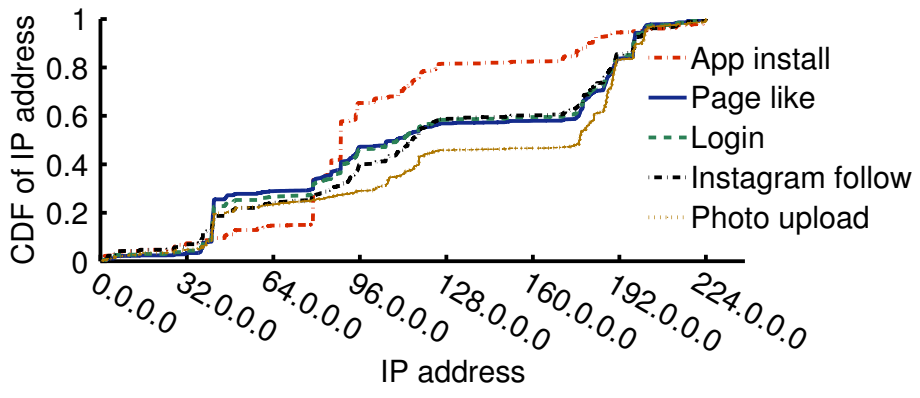

Figure 9: Distribution of the IPv4 addresses used for identified attack campaigns in each application.

dresses are administrated by large regional ISPs around the world (e.g., Global Village Telecom in Brazil and Saudi Telecom in Saudi Arabia). Some of those IP addresses are used to provide shared Internet access (e.g., for network proxies or public Internet access points). We also observed that a non-trivial fraction of the IP addresses are from hosting services such as GoDaddy and Singlehop, as well as from large cloud computing services such as Amazon AWS. This observation indicates that cloud-based services open up another avenue for threats to break into the Internet, which is in contrast to traditional botnet-based attacks [43].

\begin{tabular}{l|c|c} 
Application & $\begin{array}{c}\text { Overlap with } \\
\text { existing approaches }\end{array}$ & $\begin{array}{c}\text { New findings } \\
\text { by SynchroTrap }\end{array}$ \\
\hline Page like & $175 \mathrm{~K}$ & $555 \mathrm{~K}$ \\
\hline Instagram follow & $66 \mathrm{~K}$ & $523 \mathrm{~K}$ \\
\hline App install & N/A & $164 \mathrm{~K}$ \\
\hline Photo upload & N/A & $120 \mathrm{~K}$ \\
\hline Login & $12 \mathrm{~K}$ & $552 \mathrm{~K}$ \\
\hline Total & $253 \mathrm{~K}$ & $1,914 \mathrm{~K}$
\end{tabular}

Table 4: New findings of SynchroTrap. It uncovers a significant fraction of malicious accounts that were undetected previously. SynchroTrap is the first dedicated countermeasure in app install and photo upload at Facebook. So there is no data available from previous work to compare with.

\subsection{New findings on malicious accounts}

To evaluate SynchroTrap's capability to find malicious activities that were previously undetectable, we compare the malicious accounts detected by SynchroTrap against those detected by existing approaches inside Facebook. At Facebook, a large set of existing approaches cope with aggressive attacks by monitoring abrupt changes in certain types of user activities [35]. In each deployed application, the accounts detected by SynchroTrap in August 2013 are compared to those detected by other approaches during the same period. Table 4 shows the overlap of the malicious accounts that SynchroTrap and other approaches identified, as well as SynchroTrap's new findings. As we can see, SynchroTrap identified a large number of previously unknown malicious accounts. Specifically, in each application at least $70 \%$ of the identified accounts were not discovered by existing approaches. We investigated the exact number of accounts detected by each existing approach. We cannot report them due to confidentiality, but SynchroTrap detects a fairly large portion of those accounts. We believe that full-fledged deployment of SynchroTrap in each application on more OSN ob- 


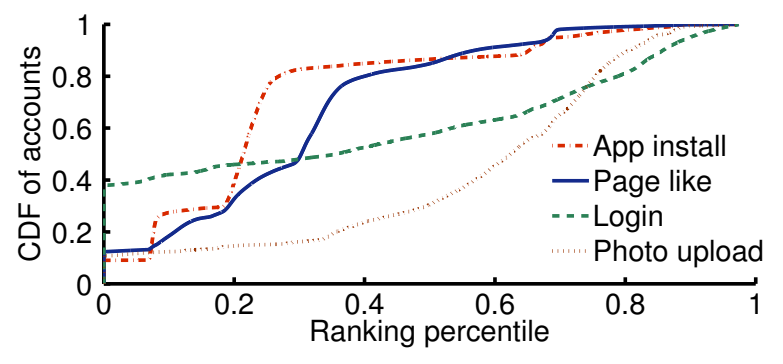

Figure 10: CDF of the detected accounts with respect to the ranking percentile generated by SybilRank. The percentiles are calculated from the bottom of the ranked list.

jects (e.g., certain fields of browser cookies) could yield more new findings and achieve higher recall of malicious accounts.

In particular, the large number of previously undiscovered malicious accounts indicates that the loosely synchronized attacks have been underestimated in existing countermeasures. SynchroTrap complements existing OSN countermeasures by effectively uncovering such attacks.

\subsection{Social connectivity of malicious accounts}

Social-graph-based defense mechanisms have attracted much attention from the research community $[19,39,41,45,46]$. We examine the social connectivity of the identified accounts by comparing them against the ranked list generated by SybilRank [19]. SybilRank discerns bulk accounts created at a low per-account cost. It ranks users based on connectivity in the social graph. Suspicious users with limited connections to legitimate users are ranked low.

We run SybilRank on a snapshot of the Facebook friendship graph obtained in August 2013. This social graph contains all Facebook users that have been perceived as benign by existing countermeasures [35] until this study. We do not include the users already stopped by existing countermeasures before the graph snapshot. Figure 10 shows the CDF of the ranking percentile of the malicious accounts that SynchroTrap detects in each Facebook application. As can be seen, a certain fraction of malicious users ( $\sim 40 \%$ in login and $\sim 15 \%$ in each of other applications) are ranked at the bottom. That portion of users are comprised of fake accounts that have little engagement on the social graph. Whereas SybilRank gives low rankings to a large portion of the identified malicious users (e.g., $80 \%$ of the detected users in app install are among the lowest $25 \%$ rankings), a non-negligible fraction of the users appear in the middle or even the top intervals of the ranked list. This indicates that attackers manipulate accounts with a variety degree of social connectivity to legitimate users. For example, a part of the accounts caught in photo upload are ranked high, presumably because attackers tend to use well-connected accounts to spread spam photos to many of their friends. As described in $\S 8.1$, these wellconnected accounts can be obtained via malware, social engineering, etc. The potential influence on the social graph and the high cost to get such accounts make them more valuable to attackers.

\subsection{Operation experience}

We perform a longitudinal study on the number of users caught by SynchroTrap for the first 11 weeks after SynchroTrap's deployment (Figure 11). From the beginning, the variation is small in Facebook login, app install, and photo upload. In contrast, the number of detected users decreases after the first month in Facebook page like and Instagram user following. It then stabilizes at around $100 \mathrm{~K}$ per week. We suspect that this drop may be caused

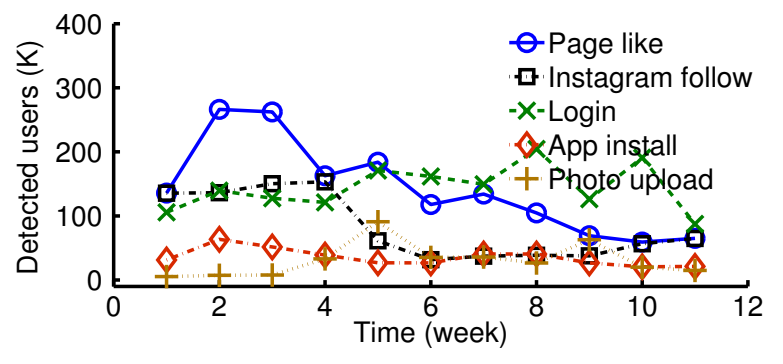

Figure 11: Number of users detected by SynchroTrap per week over a course of 11 weeks.

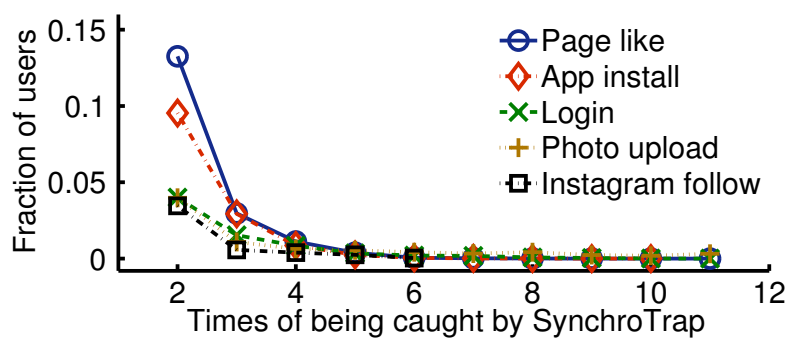

Figure 12: Distribution of the users repeatedly caught by SynchroTrap. We depict the fraction of detected users with respect to the number of times they have been repeatedly caught.

by SynchroTrap's deployment. Either the attackers are not able to obtain new controlled accounts to launch attacks or they stop the attacks temporarily to prevent their controlled accounts from being caught. The stabilized number of detected accounts in each application suggests that SynchroTrap continued to effectively detect malicious accounts over time.

Although most of the detected accounts were being caught for the first time by SynchroTrap, we observed that a non-negligible fraction of them were repeatedly caught. Figure 12 shows the fraction of these users (who were caught at least twice). As we can see, in each application 5\% 15\% of the detected users are caught twice by SynchroTrap; the fraction of users caught more than five times is less than $1 \%$. Some accounts are caught repeatedly because they are able to clear the challenges sent to them. When a malicious account is detected, Facebook's security system sends challenges such as CAPTCHAs or SMS to it. Either the attackers or the owners of the compromised accounts could have cleared the challenges so that the accounts were used to launch new attacks.

\subsection{System performance}

We evaluate the performance of SynchroTrap on a 200-machine cluster at Facebook. The daily activity data in each application is on the order of terabytes. We measure the execution time of each stage of SynchroTrap's pipeline under different parameter settings.

Daily jobs. In a daily job, the action-matching window $T_{\text {sim }}$ determines the size of the sliding comparison windows $(\S 4.5 .2)$. To examine its impact, we vary the value of $T_{\text {sim }}$ from 10 minutes to 5 hours. Figure 13 shows that the execution time of daily jobs grows as we set $T_{\text {sim }}$ to higher values. This is because a higher comparison window $T_{\text {sim }}$ causes more user pairs to be compared. As we partition data using overlapping sliding windows, each daily job in an application finishes within a few hours.

Aggregation jobs. Figure 14 shows the execution time of aggregation jobs in each application with $T_{\text {sim }}$ set to different values. 


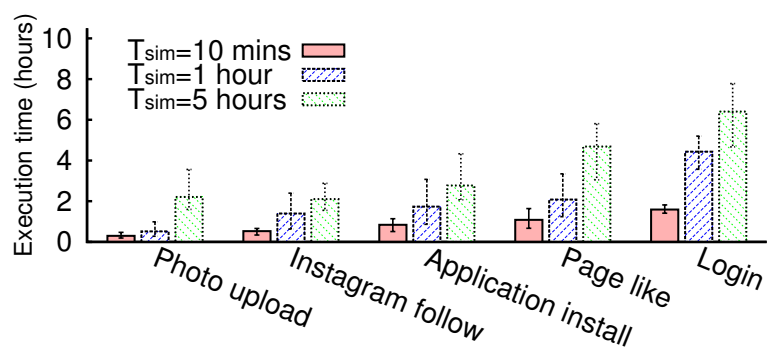

Figure 13: The execution time of SynchroTrap's daily jobs in each deployed application. We set $T_{\operatorname{sim}}$ to $10 \mathrm{mins}, 1$ hour, and 5 hours. Error bars represent $95 \%$ confident intervals.

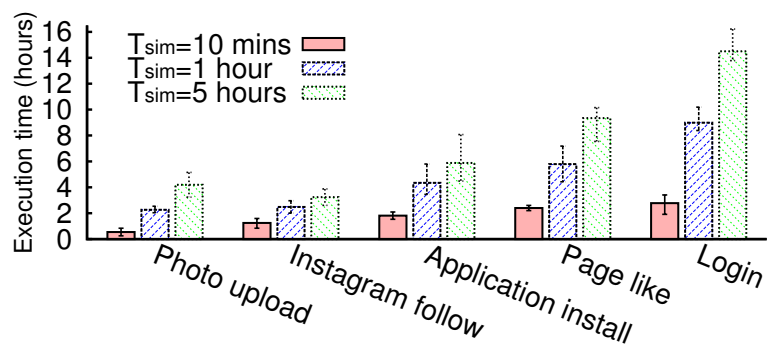

Figure 14: The execution time of aggregation jobs in each application. The input data volume varies as we generate daily user pairs using different $T_{\text {sim }}$ values (10 mins, 1 hour, and 5 hours). Error bars represent $95 \%$ confident intervals.

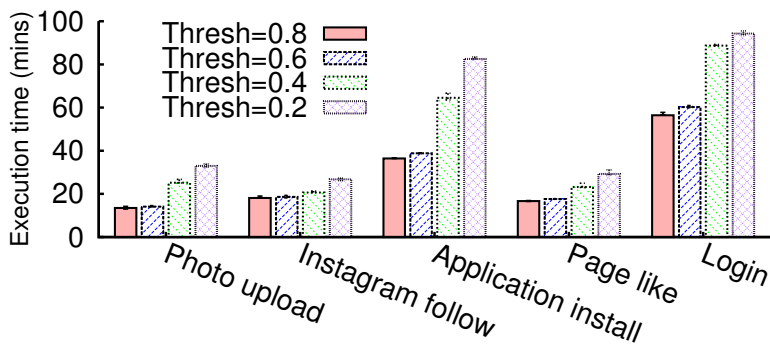

Figure 15: Execution time of finding connected components in each application. We set the similarity thresholds in our userpair filtering function to $0.2,0.4,0.6$, and 0.8. Error bars represent $95 \%$ confident intervals.

As can be seen, an aggregation job takes longer time when we increase $T_{\text {sim }}$ in the daily jobs. This is because a daily job with a larger $T_{\text {sim }}$ value generates more user pairs with matched actions, and hence increases the aggregation time. In all applications, each set of aggregation jobs completes execution within $\sim 15$ hours.

Single-linkage hierarchical clustering on Giraph. SynchroTrap's user-pair filtering function ( $\$ 4.4)$ allows distinct similarity thresholds on different granularities. We use a one-week data set to examine the execution time of clustering under varying similarity thresholds. For simplicity we assign the same value to all similarity thresholds and set this value to $0.2,0.4,0.6$, and 0.8 , respectively. Figure 15 shows that the execution time in each application increases as we set the thresholds to lower values. This is because a smaller threshold value leads to fewer user pairs to be filtered, and hence makes the user similarity graph denser. A Synchro-
Trap's clustering job finishes within $~ 100$ minutes as Giraph [11] is highly efficient.

\section{RELATED WORK}

In this section, we briefly describe previous OSN defense proposals and compare them with this work. We classify prior work into three broad categories: social-graph-based approaches, featurebased account classification, and aggregate behavior clustering. This work belongs to the category of aggregate behavior clustering.

The social-graph-based approaches $[19,46]$ use social connectivity to infer fake accounts that have limited social connections to legitimate users. They can detect a significant fraction of fake accounts that are created in bulk, but can miss well-maintained fake accounts and compromised accounts.

Feature-based account classification uses various account features to train classifiers to detect malicious accounts $[20,35,42,44]$. For example, the Facebook Immune System provides system support to manage many Facebook attack classifiers [35]. COMPA [20] identifies compromised accounts using statistical models that catch sudden changes in a user's behavior, i.e., message sending.

Clickstream [42] and CopyCatch [16] pioneered the work in aggregate behavior clustering for online social network users. Clickstream compares the pairwise similarity of the http requests from social network accounts, and clusters accounts with similar http request patterns together. It uses pre-labeled data to classify a cluster as fake or legitimate. If the number of pre-labeled fake accounts in a cluster is larger than a certain threshold, then the cluster is classified as fake; otherwise, it is legitimate. Although Clickstream achieved good detection results on a data set of $16 \mathrm{~K}$ RenRen network users, we cannot directly borrow this approach mainly because we aim to deploy SynchroTrap at much larger online social networks. First, it is practically challenging to compare all clicks from every pair of users at a large social network with hundreds of millions of active users. Second, it is difficult to obtain large volumes of training data at a large social network because it requires expensive manual labeling. Thus, many clusters may not contain any labeled data, leaving them unclassified.

CopyCatch [16], a Facebook internal system, detects fake likes casted in loose synchrony. SynchroTrap's design is based on a similar insight that malicious accounts tend to act together. However, CopyCatch assumes that a user can perform a malicious action only once (e.g., like a page at most once) and models the detection problem as a co-clustering problem [31]. When a user can repeat the same malicious action multiple times, such as log on from the same IP address repeatedly, the computational complexity of CopyCatch grows exponentially with the number of repeated actions.

In contrast, SynchroTrap assumes malicious accounts can repeat any action many times, and adopts a clustering algorithm whose computational complexity grows linearly with the number of actions an account performs ( $\$ 4.7)$. Moreover, SynchroTrap uses the source IP addresses and campaign targets to further reduce its computational complexity, making it deployable at a large-scale social network such as Facebook.

In addition to social network defense systems, SynchroTrap also borrows insight from previous work on botnet detection [21-23,43, 47], as some attackers use botnets to control malicious accounts. In particular, BotMiner [21] and BotSniffer [22] detect the bots that respond to commands in a similar way. BotGraph [47] detects botnet IP addresses that are shared by a large number of spamming email accounts. SynchroTrap also uses shared IP addresses as a signal to detect groups of malicious accounts, but uses the timestamps of user actions to further improve detection accuracy. 


\section{CONCLUSION}

This work aims to detect large groups of active malicious accounts in OSNs, including both fake accounts and compromised real user accounts. We designed a generic and scalable detection system, SynchroTrap, that uses clustering analysis to detect large groups of malicious users that act in loose synchrony. To cope with the enormous volume of user activity data in a large OSN, we implemented SynchroTrap as an incremental processing system on top of Hadoop and Giraph. We further optimize it by partitioning user activity data by time and only comparing pair-wise user actions that fall into overlapping sliding windows. We deployed SynchroTrap in five applications at Facebook and Instagram. During one month of deployment, SynchroTrap unveiled 1156 large campaigns and more than two million malicious accounts that involved in the campaigns.

Although we designed SynchroTrap for OSNs, we believe that the approach of detecting loosely synchronized actions can also uncover large attacks in other online services, such as web email and electronic commerce, at the present time. Furthermore, the incremental processing and data partitioning techniques we have explored may benefit other applications that analyze large volume of time-independent data by reducing the requirements on their computing infrastructure.

Finally, we note that SynchroTrap's design uses unsupervised learning and does not detect malicious actions in real time. In the future, we can extract attack signatures from the malicious campaigns and accounts it detects and use supervised learning to develop fast classifiers that can detect attacks in real time.

\section{ACKNOWLEDGMENTS}

We thank Yuchun Tang and the anonymous reviewers for their valuable suggestions. We are grateful to Matt Jones, Benjamin Yang, Abe Land, Ioannis Papagiannis, and many other members from the Facebook Site Integrity team for their help during this project. We are particularly thankful to Michael Sirivianos for his extensive feedback. We also thank the Facebook Digraph team for providing the graph processing infrastructure. This work was supported in part by NSF Awards CNS-0845858 and CNS-1017858.

\section{REFERENCES}

[1] Better Security through Software. http://www.facebook.com/notes/ facebook/better-security-through-sof tware/248766257130, 2010.

[2] Staying in Control of Your Facebook Logins. http://www.facebook.com/ notes/facebook/staying-in-control-of-your-facebook-logins/ 389991097130, 2010.

[3] Working Together to Keep You Secure. http://www.facebook.com/notes/ facebook/working-together-to-keep-you-secure/68886667130, 2010.

[4] Amazon EC2 Pricing. http://aws .amazon.com/ec2/pricing/, 2013.

[5] Apache Giraph. http://giraph.apache.org/, 2013.

[6] Apache Hadoop. http://hadoop.apache.org/, 2013

[7] Cookies, Pixels \& Similar Technologies. https://www.facebook.com/ help/cookies, 2013.

[8] Facebook Reports Fourth Quarter and Full Year 2012 Results. http: // investor.fb.com/releasedetail. cfm?ReleaseID=736911, 2013

[9] Facebook's New Way to Combat Child Pornography. http://gadgetwise. blogs.nytimes.com/2011/05/19/facebook-to-combat-child-pornusing-microsofts-technology, 2013.

[10] Malicious Chrome extensions on the rise. http://www.zdnet.com/ malicious-chrome-extensions-on-the-rise-7000019913/, 2013.

[11] Scaling Apache Giraph to a trillion edges. https ://www.facebook.com/ notes/facebook-engineering/scaling-apache-giraph-to-atrillion-edges/10151617006153920, 2013.

[12] Types of cookies used by Google. http://www.google.com/policies/ technologies/types/, 2013.

[13] Rate Limiting at Facebook. https://developers.facebook.com/docs/ reference/ads-api/api-rate-limiting, 2014.

[14] Rate Limiting at Google+. https://developers.google.com/+/ domains/faq, 2014.
[15] I. Anderson. Combinatorics of finite sets. Clarendon Press, 1987.

[16] A. Beutel, W. Xu, V. Guruswami, C. Palow, and C. Faloutsos. CopyCatch: Stopping Group Attacks by Spotting Lockstep Behavior in Social Networks. In Proceedings of the 22nd International Conference on World Wide Web (WWW), 2013.

[17] L. Bilge, T. Strufe, D. Balzarotti, and E. Kirda. All Your Contacts Are Belong to Us: Automated Identity Theft Attacks on Social Networks. In WWW, 2009.

[18] F. Brunk. Intersection Problems in Combinatorics. University of St Andrews thesis. University of St Andrews, 2009.

[19] Q. Cao, M. Sirivianos, X. Yang, and T. Pregueiro. Aiding the Detection of Fake Accounts in Large Scale Social Online Services. In NSDI, 2012.

[20] M. Egele, G. Stringhini, C. Krügel, and G. Vigna. COMPA: Detecting Compromised Accounts on Social Networks. In NDSS, 2013.

[21] G. Gu, R. Perdisci, J. Zhang, and W. Lee. BotMiner: Clustering Analysis of Network Traffic for Protocol- and Structure-Independent Botnet Detection. In USENIX SECURITY, 2008.

[22] G. Gu, J. Zhang, and W. Lee. BotSniffer: Detecting Botnet Command and Control Channels in Network Traffic. In NDSS, 2008.

[23] S. Hao, N. A. Syed, N. Feamster, A. G. Gray, and S. Krasser. Detecting Spammers with SNARE: Spatio-Temporal Network-Level Automatic Reputation Engine. In USENIX SECURITY, 2009.

[24] P. Jaccard. The Distribution of the Flora in the Alpine Zone. New Phytologist, 11(2), 1912.

[25] T. N. Jagatic, N. A. Johnson, M. Jakobsson, and F. Menczer. Social Phishing. Communications of the ACM, 50(10), 2007.

[26] A. K. Jain, M. N. Murty, and P. J. Flynn. Data Clustering: a Review. ACM Computing Surveys, 31(3), 1999.

[27] U. Kang, C. E. Tsourakakis, and C. Faloutsos. PEGASUS: Mining Peta-Scale Graphs. Knowl. Inf. Syst., 27(2), 2011

[28] G. Karypis and V. Kumar. Multilevel Algorithms for Multi-Constraint Graph Partitioning. In Proceedings of the 1998 ACM/IEEE conference on Supercomputing, 1998.

[29] H.-P. Kriegel, P. Kröger, and A. Zimek. Clustering High-Dimensional Data: A Survey on Subspace Clustering, Pattern-Based Clustering, and Correlation Clustering. ACM Trans. Knowl. Discov. Data, 3(1), 2009.

[30] G. Malewicz, M. H. Austern, A. J. Bik, J. C. Dehnert, I. Horn, N. Leiser, and G. Czajkowski. Pregel: a System for Large-Scale Graph Processing. In SIGMOD, 2010.

[31] I. V. Mechelen, H. H. Bock, and P. D. Boeck. Two-mode clustering methods: a structured overview. Statistical Methods in Medical Research, 13:363-394, 2004.

[32] D. Moore, C. Shannon, G. M. Voelker, and S. Savage. Internet Quarantine: Requirements for Containing Self-Propagating Code. In INFOCOM, 2003.

[33] M. Motoyama, D. McCoy, K. Levchenko, S. Savage, and G. M. Voelker. Dirty Jobs: the Role of Freelance Labor in Web Service Abuse. In USENIX SECURITY, 2011

[34] R. Nishtala, H. Fugal, S. Grimm, M. Kwiatkowski, H. Lee, H. C. Li, R. McElroy, M. Paleczny, D. Peek, P. Saab, D. Stafford, T. Tung, and V. Venkataramani. Scaling Memcache at Facebook. In NSDI, 2013.

[35] T. Stein, E. Chen, and K. Mangla. Facebook Immune System. In Proceedings of the 4th Workshop on Social Network Systems (SNS), 2011.

[36] K. Thomas, C. Grier, D. Song, and V. Paxson. Suspended accounts in retrospect: An analysis of twitter spam. In IMC, 2011.

[37] K. Thomas, D. McCoy, C. Grier, A. Kolcz, and V. Paxson. Trafficking Fraudulent Accounts: The Role of the Underground Market in Twitter Spam and Abuse. In USENIX SECURITY, 2013.

[38] A. Thusoo, Z. Shao, S. Anthony, D. Borthakur, N. Jain, J. Sen Sarma, R. Murthy, and H. Liu. Data Warehousing and Analytics Infrastructure at Facebook. In SIGMOD, 2010.

[39] D. N. Tran, B. Min, J. Li, and L. Subramanian. Sybil-Resilient Online Content Rating. In NSDI, 2009.

[40] C. E. Tsourakakis, C. Gkantsidis, B. Radunovic, and M. Vojnovic. Fennel: Streaming Graph Partitioning for Massive Scale Graphs. Microsoft Technical Report MSR-TR-2012-113, 2012.

[41] B. Viswanath, A. Post, K. P. Gummadi, and A. Mislove. An Analysis of Social Network-based Sybil Defenses. In SIGCOMM, 2010.

[42] G. Wang, T. Konolige, C. Wilson, X. Wang, H. Zheng, and B. Y. Zhao. You are How You Click: Clickstream Analysis for Sybil Detection. In USENIX SECURITY, 2013.

[43] Y. Xie, F. Yu, K. Achan, R. Panigrahy, G. Hulten, and I. Osipkov. Spamming Botnets: Signatures and Characteristics. In SIGCOMM, 2008.

[44] Z. Yang, C. Wilson, X. Wang, T. Gao, B. Y. Zhao, and Y. Dai. Uncovering Social Network Sybils in the Wild. In IMC, 2011.

[45] H. Yu, P. Gibbons, M. Kaminsky, and F. Xiao. SybilLimit: A Near-Optimal Social Network Defense Against Sybil Attacks. In IEEE S\&P, 2008.

[46] H. Yu, M. Kaminsky, P. B. Gibbons, and A. Flaxman. SybilGuard: Defending Against Sybil Attacks via Social Networks. In SIGCOMM, 2006.

[47] Y. Zhao, Y. Xie, F. Yu, Q. Ke, Y. Yu, Y. Chen, and E. Gillum. BotGraph: Large Scale Spamming Botnet Detection. In NSDI, 2009.

[48] C. C. Zou, W. Gong, and D. Towsley. Worm Propagation Modeling and Analysis Under Dynamic Quarantine Defense. In Proceedings of the 2003 ACM Workshop on Rapid Malcode (WORM), 2003. 